\title{
The Soundless Outcry
}

\author{
Su Kyoung An, Hea Joo Lee, \& Jiang Li \\ Central Michigan University
}

Keywords: Declaration, Korean independence, digital printing, laser cutting

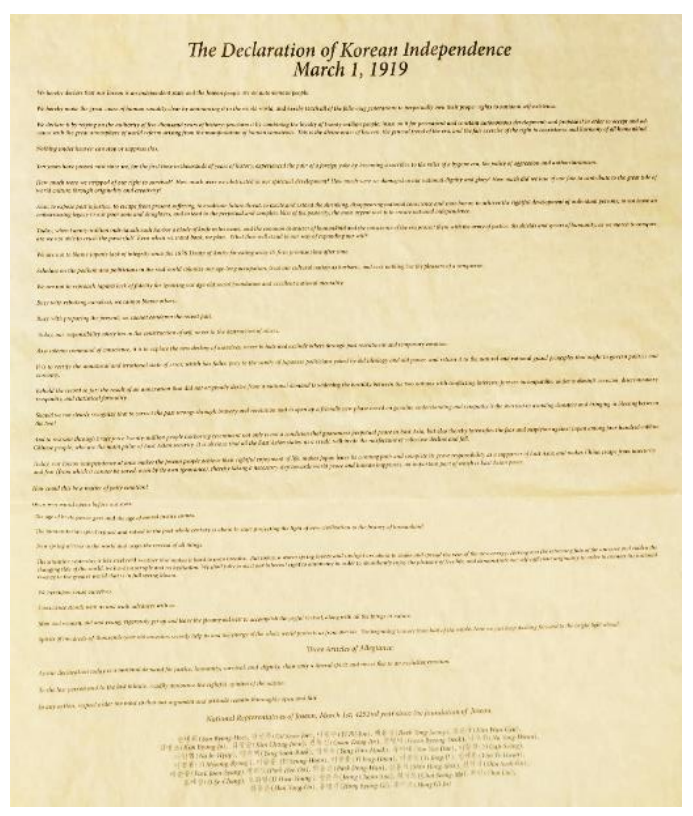

Figure 1. Declaration of Korean Independence
The Soundless Outcry was an ensemble that was inspired by the Declaration of Korean Independence (Figure 1), which was announced by 33 ethnic representatives in Korea on March 1919, for Korea's independence (Kim, 2019). In 1919, Korea was under Japanese colonial rule for nine years. Some intellectual Koreans secretly prepared the Declaration of Independence for Korea's independence (Ch'oe, Lee, \& Bary, 2000). They announced that Korea would no longer tolerate Japanese colonial rule through the Declaration of Korea Independence on March $1^{\text {st }}, 1919$ in public (Kim, 2019). This announcement was the largely peaceful speech in the world and followed more than a million Koreans' non-violent and democratic uprising. It also became the spark of the March $1^{\text {st }}$ civil movement that led Korea to independence. Nearly thirty years later, Korea gained independence after the defeat of the Empire of Japan in World War II (Ch'oe, Lee, \& Bary, 2000). As the design scholars, The Soundless Outcry was designed to commemorate 100 years of March $1^{\text {st }}$ Movement and to remind the importance and meaning of Korean's non-violent and democratic uprising through the Declaration of Korean Independence.

This wearable art piece primarily emphasizes the traditional Korean dress as to embody the spirit of the Koreans at the time of oppression during the colonization of Korea by Japan during World War II. The biggest consideration that went into this ensemble was to showcase both the fighting spirit of the Koreans and the hardship the circumstances brought to the Korean people during this period.

The silhouette of this wearable art piece was inspired by the traditional Korean male outfits, a top and a bottom called jeogori and baji, by adding shape and volume to both. The pattern pieces for both were flat-patterned with three times adjustments. All pieces were then digitized into

Page 1 of 4

Published under a Creative Commons Attribution License (https://creativecommons.org/licenses/by/4.0/), which permits unrestricted use, distribution, and reproduction in any medium, provided the original work is properly cited.

ITAA Proceedings, \#76 - https://itaaonline.org 
Modaris and converted into DXF files to be opened in Adobe Illustrator for applying the textile motifs, which was inspired from Declaration of Korean Independence.

The designers selected seven most important and meaningful sentences from the Declaration of Korean Independence and both English and Korean versions of these sentences were modified to engineer into the patterns. In the front and back flare bodices, the stovepipe neckline was designed with gold bindings to symbolize the pride and indomitable will the Koreans showed against the persecution of the Japanese during the colonization. Also, the wide bell sleeves were created by using a weaving technique constructed in a way that highlights the turbulent period of Korea's history by weaving irregularly with the sentence straps. The $1 \frac{1 / 4}{4}$ straps patterns were engineered with the selected seven sentences (both English and Korean versions) in Adobe Illustrator. The engineered straps patterns were digitally printed on the poly-blend taffeta fabric (Figure 2). The seven sentences were also placed into the front and back pant patterns (Figure 3) for engineered garment and digital printed on the same poly-blend taffeta fabric. The color of the pants and straps on the sleeve was created with a smooth gradient from black to light yellow. It was intended to express the "Hope" that the citizens of Korea had at the time for the independence and freedom from the dark age of colonization.

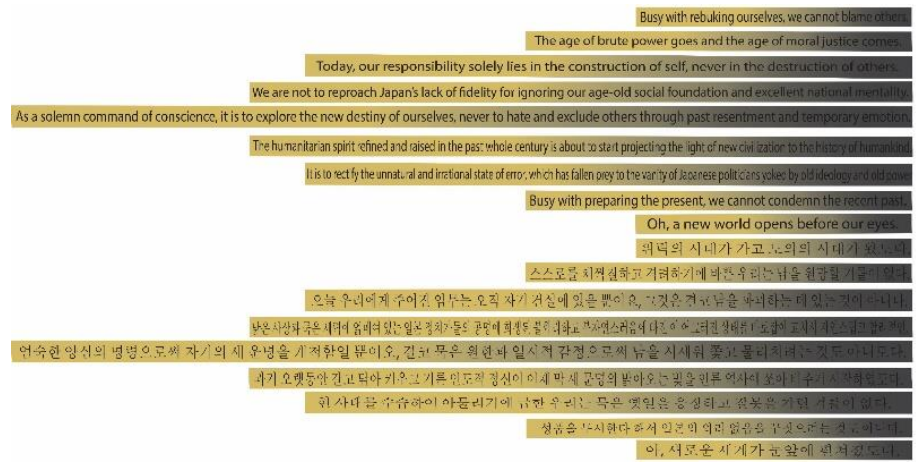

Figure 2. Strap Patterns

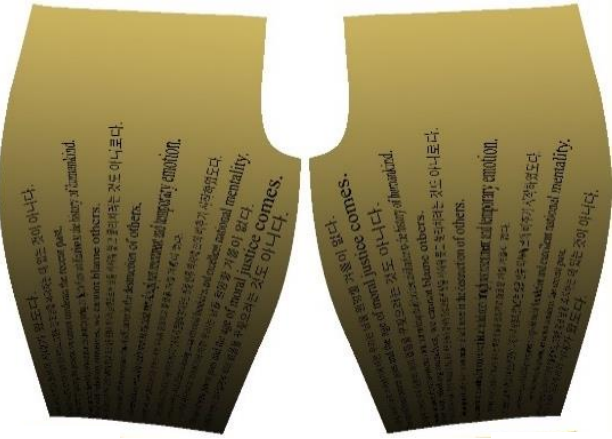

Figure 3. Front and Back Pant Patterns

The Universal Laser ILS 12.150D model was utilized. The designers adjusted the power and speed of the laser-cutter system to fuse the cutting edges and prevent the fabric from fraying and raveling. Through several tests, the appropriate settings for the cutting power, speed, and PPI were found. The front and back pants, waist cuff, ankle cuff, waist long strap, and sleeve straps were laser-cut with an $80 \%$ power, $100 \%$ speed, and 1000 PPI on the poly-blend digital printed taffeta. The front and back flare bodice, and wide bell sleeve were laser-cut with a $90 \%$ power, $100 \%$ speed, and 1000 PPI on the black polyester sateen.

All details which applied to the outfits were also inspired from the traditional Korean costume. The 4" wide cuffs were designed to the waist and ankles to interpret the traditional Korean pants'

Published under a Creative Commons Attribution License (https://creativecommons.org/licenses/by/4.0/), which permits unrestricted use, distribution, and reproduction in any medium, provided the original work is properly cited.

ITAA Proceedings, \#76 - https://itaaonline.org 
details. Two black knot buttons were also applied to each ankle cuff on the pant. On the waist, the 4" wide long strap was tied in the same way as the traditional Korean style. For top closure, the black invisible zipper was attached on the center back seam with the gold bindings. Two hooks and eyes were applied on the right side of pant.

The Soundless Outcry described Korea's non-violent and democracy struggle that led Korea to independence and expressed the meaning of the Declaration of Korean Independence. Through this work, the designers would like to show how much Koreans aspired to independence from the Japanese colonial rule and how much they yearned for freedom.

\section{References:}

Ch'oe, Y., Lee, P., \& Bary, W. (2000). Declaration of Independence (March 1st, 1919). New York, NY: Columbia University Press.

Kim, R. (2019). Korea's March First movement of 1919, 100 years later. Retrieved from https://americanpyongyang.com/2019/03/01/koreas-march-first-movement-of-1919-100years-later/ 


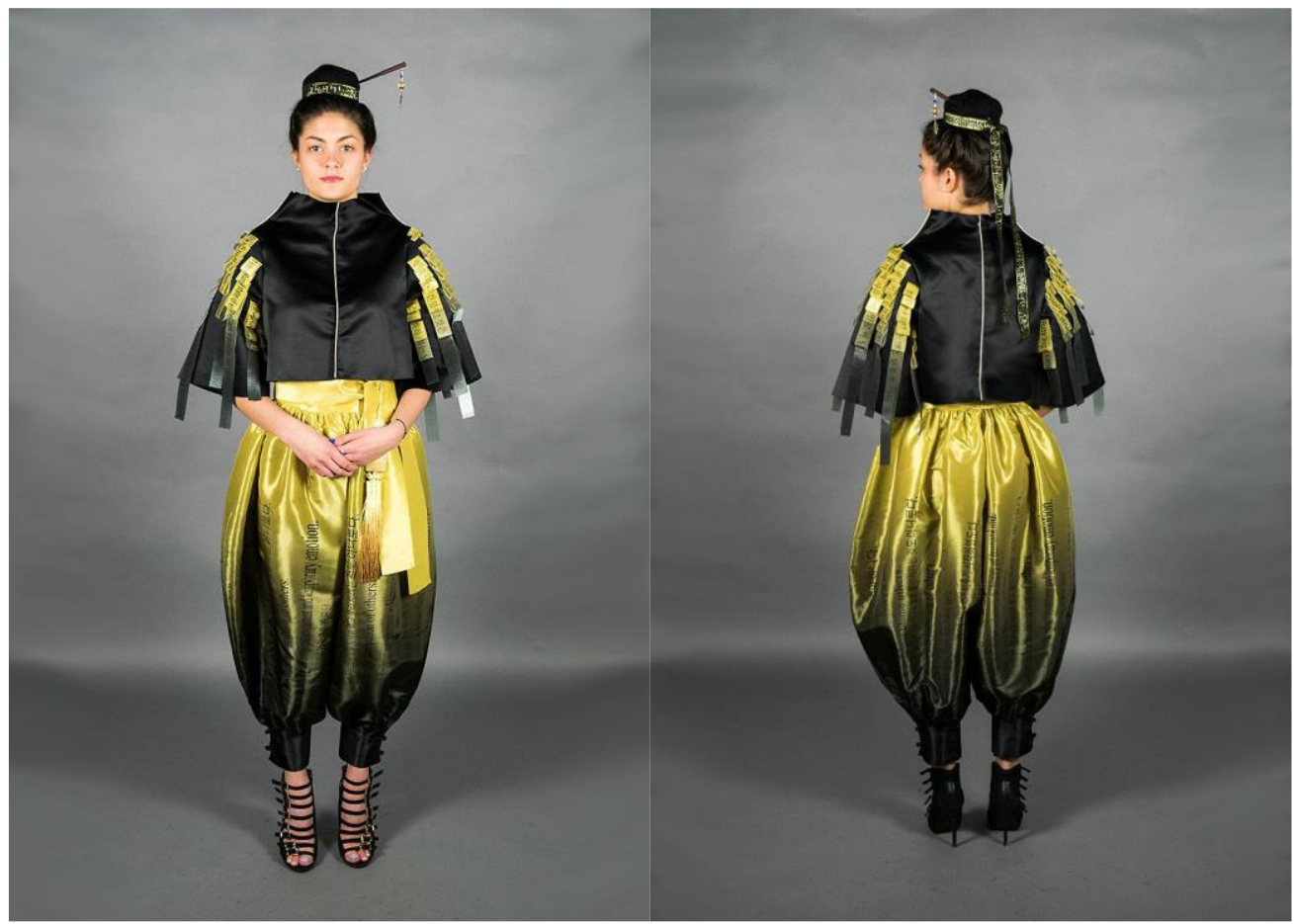

Image A. Front View

Image B. Back View

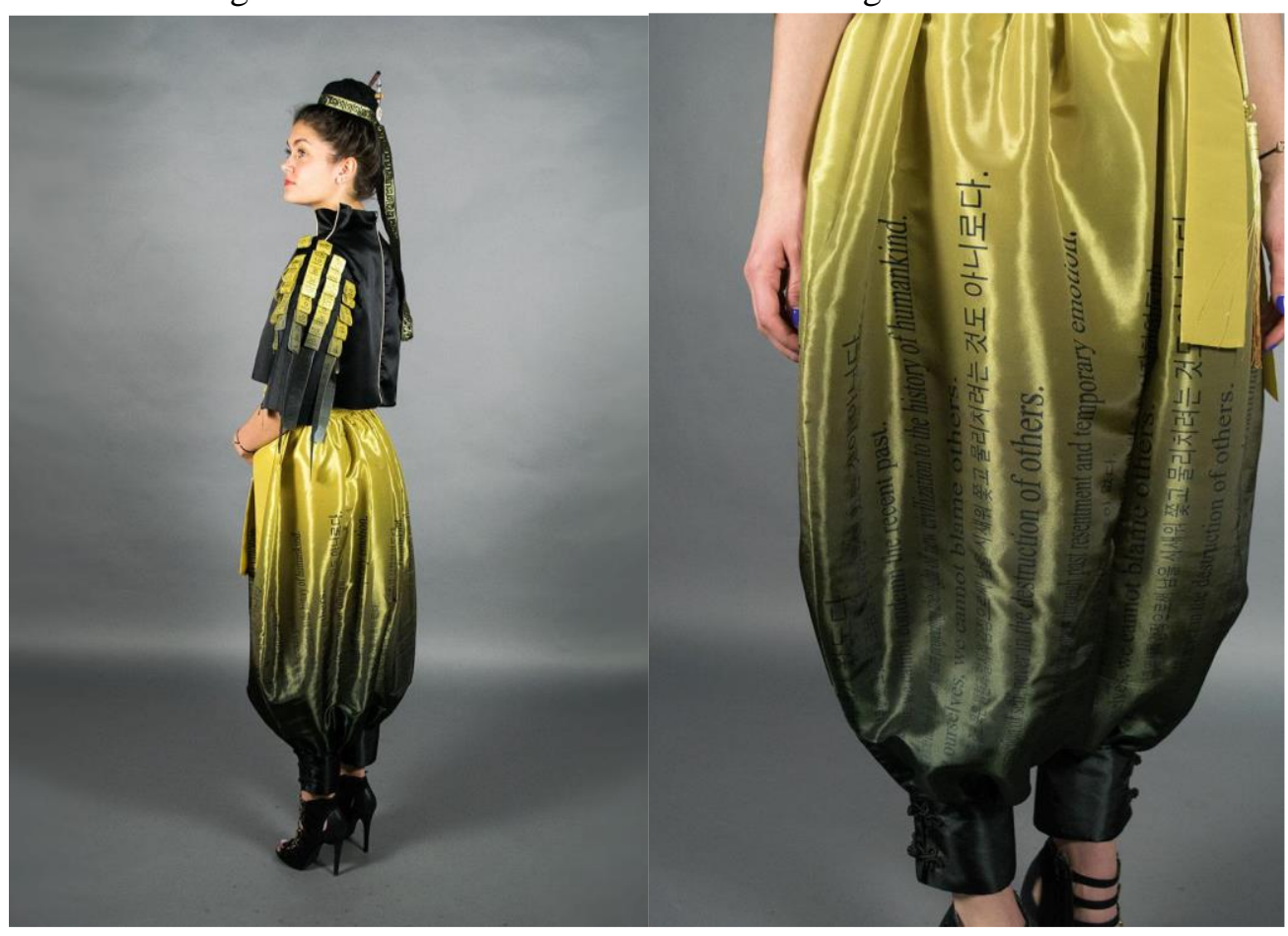

Image C. Side View

Image D. Detail View

Page 4 of 4

Published under a Creative Commons Attribution License (https://creativecommons.org/licenses/by/4.0/), which permits unrestricted use, distribution, and reproduction in any medium, provided the original work is properly cited.

ITAA Proceedings, \#76 - https://itaaonline.org 